\title{
Hyperbolic self avoiding walk
}

\author{
Itai Benjamini* Christoforos Panagiotis ${ }^{\dagger}$
}

\begin{abstract}
Ballisticity of a continuous self avoiding walk on hyperbolic spaces $\mathbb{H}^{d}$ is established.

Keywords: self avoiding walk; hyperbolic space; convex hull. MSC2020 subject classifications: $05 \mathrm{C} 81 ; 51 \mathrm{M} 10$.

Submitted to ECP on August 15, 2020, final version accepted on March 18, 2021.
\end{abstract}

\section{Introduction}

Consider the continuous $n$-step random walk on the hyperbolic $d$-space, denoted $\mathbb{H}^{d}$, where the next step is chosen uniformly and independently on the $(d-1)$-dimensional sphere around the current location. Condition this uniform product measure on sequences in which the distance between any pair of vertices is bigger than $c$, for some fixed $0<c<1$.

We will write $\mathbb{P}_{n}$ for this measure, which will be called the SAW measure, and $\mathbb{E}_{n}$ for the expectation with respect to this measure. We denote by $x_{0}, x_{1}, \ldots, x_{n}$ the vertices of the walk in order of appearance and $d(\cdot, \cdot)$ the distance function.

Theorem 1.1. There is a constant $C=C(d, c)>0$ such that $\mathbb{E}_{n} d\left(x_{0}, x_{n}\right)>C n$ for every $n \geq 1$.

For background on hyperbolic geometry see e.g. [4]. For background on self avoiding walks see [1]. The papers $[2,5,7,9,8,10]$ contain results on the speed of self avoiding walk.

The self avoiding walk model has been primarily studied on graphs. The most wellstudied cases are the hypercubic lattice $\mathbb{Z}^{d}$ and other Euclidean lattices. In this setting, the self avoiding walk is known to be sub-ballistic and the model is well-understood in dimensions $d \geq 5[5,6]$. Recently, the study of self avoiding walk on non-Euclidean lattices has received increasing attention. Madras and Wu [9] proved that the SAW is ballistic for some regular tessellations of the hyperbolic plane of large enough degree. The second author [10] extended this result to all regular tessellations of the hyperbolic plane. The main idea of the latter paper is to use percolation on the underlying graph as a tool for obtaining upper bounds for the number of 'closed' self avoiding walks of length $n$, namely self avoiding polygons. This method utilises the fact that in 2 dimensions, a self avoiding polygon encloses a bounded region, to associate to each self avoiding polygon a notion of inner boundary. Then a certain isoperimetric inequality is obtained which compares the length of a self avoiding polygon with the size of its inner boundary.

*Weizmann Institute of Science, Israel. E-mail: itai.benjamini@gmail.com

${ }^{\dagger}$ Université de Genève, Switzerland. E-mail: christoforos.panagiotis@unige.ch 
Hyperbolic self avoiding walk

A direct counting argument provides lower bounds for the total number of self avoiding walks of length $n$, and proves that the self avoiding walk is ballistic. See [7, 8] for more results on the speed of self avoiding walk on non-Euclidean lattices.

The aforementioned argument makes strong use of planarity and the combinatorial structure of the model in the graph case, and it is not clear how to modify it in order to make it work in our context. Our approach is more geometric in nature, and the current paper replaces a paper [2] regarding self avoiding walk on the 7-regular triangulation of the hyperbolic plane by the first author. Since our space has constant negative curvature, for every self avoiding walk $x$, a constant proportion of its points are close to the boundary of the convex hull of $x$. The abundance of local symmetries allows us to modify the trajectory of $x$ between $x_{i}$ and $x_{n}$, where $x_{i}$ is a point close to the boundary of the convex hull, to obtain a self avoiding walk $x^{\prime}$ such that $x_{i}^{\prime}$ is at bounded distance from the geodesic between $x_{0}^{\prime}$ and $x_{n}^{\prime}$. As it turns out, this local modification happens with probability bounded away from 0 , and allows us to obtain that in expectation, a constant proportion of vertices of $x$ are at bounded distance from the geodesic between $x_{0}$ and $x_{n}$. This implies that the length of the geodesic is proportional to $n$, as desired.

After proving the main result of this paper, we mention some open problems regarding the behaviour of the model once we make the steps of the walk tend to 0 . These problems are motivated by related open problems regarding self avoiding walk on $\mathbb{R}^{d}$.

\section{Proof}

Proof. Let us first recall that there is a constant $\delta>0$ fixed throughout such that for every geodesic triangle in $\mathbb{H}^{d}$ with sides $\alpha, \beta$, $\gamma$, we have that $\alpha$ lies in the $\delta$ neighbourhood of $\beta \cup \gamma$.

We will start by proving the following geometric lemma.

Lemma 2.1. There is a constant $c_{1}>0$ such that for every finite set $A$ of vertices in $\mathbb{H}^{d}$ with the property that the distance between any two of its points is at least $c$, at least $c_{1}|A|$ vertices of $A$ are at distance at most $(1-c) / 2$ from the boundary of the convex hull of $A$.

Proof. Notice that the convex hull $K$ of $A$ coincides with the convex hull of $A \cap \partial K$. According to [3], there is a constant $t=t(d)$ such that the volume of $K$ is at most $t|A \cap \partial K|$. For every $x$ in $B:=\{x \in A \mid d(x, \partial K)>(1-c) / 2\}$, the ball of radius $(1-c) / 2$ around $x$ is contained in $K$, hence the volume of $K$ is greater than $t^{\prime}|B|$ for a certain constant $t^{\prime}>0$. The assertion follows now easily.

For a set of vertices $A$, we will write $\mathcal{H}(A)$ for the convex hull of $A$. It follows from Lemma 2.1 that for every large enough $M>0$ and for every SAW $x$ of length $n$, the number of indices $i$ with $d\left(x_{i}, \partial \mathcal{H}(x)\right) \leq(1-c) / 2$ and $d\left(x_{j}, \partial \mathcal{H}(x)\right) \leq(1-c) / 2$ for some $i+1 \leq j \leq i+M$ is proportional to $n$. As there are finitely many choices for $j-i$, there is some $1 \leq r=r(x) \leq M$ such that the number of indices $i$ with $d\left(x_{i}, \partial \mathcal{H}(x)\right) \leq(1-c) / 2$ and $d\left(x_{i+r}, \partial \mathcal{H}(x)\right) \leq(1-c) / 2$ is proportional to $n$. In fact, we can assume that $r$ is as large as we want at the expense of possibly increasing $M$ and reducing the constant of proportionality. In particular, we can always choose $r>3 \delta+1-c$. We fix an $M>0$ for which this is always possible. The reason for making this choice will become clear later.

For any number $\mathcal{C}>0$, we define $\mathcal{A}_{i}(\mathcal{C})$ to be the event that $x_{i}$ has distance at most $\mathcal{C}$ from the geodesic between $x_{0}$ and $x_{n}$. We also define $\mathcal{B}_{i, r}$ to be the event that $d\left(x_{i}, \partial \mathcal{H}(x)\right) \leq(1-c) / 2$ and $d\left(x_{i+r}, \partial \mathcal{H}(x)\right) \leq(1-c) / 2$, and we let $\mathcal{B}_{i}$ be the event that $\mathcal{B}_{i, r}$ occurs for some $r$ such that $M \geq r>3 \delta+1-c$. Our aim is to utilize the above observation in order to modify the SAWs of $\mathcal{B}_{i}$ to construct SAWs that satisfy $\mathcal{A}_{i}(\mathcal{C})$. 
In order to do this, we would like to use some simple facts of hyperbolic geometry that are easy to see once we fix a model. Let us consider the Poincaré ball model for $\mathbb{H}^{d}$, which is defined as the unit ball in $\mathbb{R}^{d}$ equipped with the following metric:

$$
d s^{2}=4 \frac{d x_{1}^{2}+\ldots+d x_{d}^{2}}{\left(1-x_{1}^{2}-\ldots-x_{d}^{2}\right)^{2}} .
$$

We recall that geodesics consist of all arcs of Euclidean circles contained within the unit ball that are orthogonal to the boundary of the unit ball, plus all diameters of the unit ball. As a consequence, a hyperbolic hyperplane lies either in a Euclidean sphere or a Euclidean hyperplane passing through the origin. Moreover, a hyperbolic ball is also a Euclidean ball. The hyperbolic and Euclidean centres are, in general, not the same, but they coincide in case one of them is the origin. The model has also the advantage of being conformal, i.e. hyperbolic angles coincide with Euclidean angles.

In the following lemma, we will write $\overline{x y}$ for the geodesic between points $x$ and $y$ in $\mathbb{H}^{d}$ and $d(A, B):=\inf _{x \in A, y \in B} d(x, y)$ for the distance between subsets of $\mathbb{H}^{d}$.

Lemma 2.2. There are universal constants $0<C<1, \mathcal{C}>0$ such that $\mathbb{P}_{n}\left(\mathcal{A}_{i}(\mathcal{C})\right) \geq$ $C \mathbb{P}_{n}\left(\mathcal{B}_{i}\right)$ for every large enough $n$ and every $i \geq 1$.

Proof. Consider some $M \geq r>3 \delta+1-c$ and a SAW $x \in \mathcal{B}_{i, r}$. Our aim is to define a family of walks $x^{\prime}$ for which the event $\mathcal{A}_{i}(\mathcal{C})$ occurs for a certain constant $\mathcal{C}>0$, while $\left\{x_{0}^{\prime}, \ldots, x_{i}^{\prime}\right\}$ coincides with $\left\{x_{0}, \ldots, x_{i}\right\}$, and $\left\{x_{i+r}^{\prime}, \ldots, x_{n}^{\prime}\right\}$ coincides with the image of $\left\{x_{i+r}, \ldots, x_{n}\right\}$ under an isometry of $\mathbb{H}^{d}$. We will say that $\left\{x_{i+r}^{\prime}, \ldots, x_{n}^{\prime}\right\}$ and $\left\{x_{i+r}, \ldots, x_{n}\right\}$ have the same relative position.

To this end, let $z_{i}, z_{i+r}$ be the points in the boundary of $\mathcal{H}\left(x_{0}, \ldots, x_{i}\right), \mathcal{H}\left(x_{i+r}, \ldots, x_{n}\right)$ closest to $x_{i}, x_{i+r}$, respectively. Consider two separating hyperplanes $H_{1}$ and $H_{2}$ that pass through $z_{i}$ and $z_{i+r}$, respectively. Notice that $H_{1}$ and $H_{2}$ divide $\mathbb{H}^{d}$ into two half spaces. Let $S_{1}$ be the half space with $\partial S_{1}=H_{1}$ that contains $\mathcal{H}\left(x_{0}, \ldots, x_{i}\right)$ and $S_{2}$ be the half space with $\partial S_{2}=H_{2}$ that contains $\mathcal{H}\left(x_{i+r}, \ldots, x_{n}\right)$.

Consider an isometry $f$ of $\mathbb{H}^{d}$ such that the distance between $z_{i}$ and $f\left(z_{i+r}\right)$ is equal to

$$
m:=r-d\left(x_{i}, z_{i}\right)-d\left(x_{i+r}, z_{i+r}\right) \geq r+c-1>3 \delta .
$$

We can find a rotation $g$ around $f\left(z_{i+r}\right)$ so that the geodesic connecting $z_{i}$ to $(g \circ f)\left(z_{i+r}\right)$ is normal to the hyperplane $(g \circ f)\left(H_{2}\right)$. Then we can find a rotation $h$ around $z_{i}$ so that the geodesic connecting $z_{i}$ to $T\left(z_{i+r}\right)$ is normal to the hyperplane $H_{1}$, where $T:=h \circ g \circ f$, and at the same time, $S_{1}$ and $T\left(S_{2}\right)$ are disjoint. We claim that $d\left(S_{1}, T\left(S_{2}\right)\right)=d\left(z_{i}, T\left(z_{i+r}\right)\right)=$ $m$. Indeed, the distance $d\left(S_{1}, T\left(S_{2}\right)\right)$ is attained by some $p_{0} \in S_{1}, q_{0} \in T\left(S_{2}\right)$, because the Euclidean distance of $S_{1}$ and $T\left(S_{2}\right)$ in our model is positive, hence the hyperbolic distance $d(p, q), p \in S_{1}, q \in T\left(S_{2}\right)$ becomes larger than $m$ when $p$ or $q$ are close enough (in the Euclidean sense) to the ideal boundary of our model. The hyperbolic ball of radius $d\left(S_{1}, T\left(S_{2}\right)\right)$ around $p_{0}$ must contain only one point of $T\left(S_{2}\right)$, hence $\overline{p_{0} q_{0}}$ is normal to $T\left(S_{2}\right)$. Similarly, $\overline{p_{0} q_{0}}$ is normal to $S_{1}$. Unless $p_{0}=z_{i}$ and $q_{0}=T\left(z_{i+r}\right)$, the points $p_{0}, q_{0}, z_{i}, T\left(z_{i+r}\right)$ define either a quadrilateral with angles that add up to $2 \pi$ or a triangle with angles that add up to a number larger than $\pi$. This is impossible in a hyperbolic space, hence $p_{0}=z_{i}$ and $q_{0}=T\left(z_{i+r}\right)$. This proves the claim.

Notice that $d\left(x_{i}, T\left(x_{i+r}\right)\right)=r$. Consider now the geodesic walk $y_{i}=x_{i}, y_{i+1}, \ldots$, $y_{i+r}=T\left(x_{i+r}\right)$ of length $r$ that connects $x_{i}$ to $T\left(x_{i+r}\right)$, where $d\left(y_{j}, y_{j+1}\right)=1$ for every $j=i, \ldots, i+r-1$. Adding this geodesic walk to $x_{0}, \ldots, x_{i}$ and $T\left(x_{i+r}\right), \ldots, T\left(x_{n}\right)$ results in a walk $x^{\prime}$ of length $n$. Notice that this walk is self avoiding because each $y_{i+1}, \ldots, y_{i+r-1}$ is at distance at least $1-(1-c) / 2>c$ from both $S_{1}$ and $T\left(S_{2}\right)$.

Modifying slightly the steps of $x^{\prime}$, we can construct a lot of SAWs with the desired properties. Indeed, let $k:=\min \left\{\frac{1-c}{2}, \frac{r+c-1-3 \delta}{2}\right\}$. Let $W$ be the set of walks $w_{i+1}, \ldots, w_{i+r}$ 
for which $d\left(w_{i+1}, y_{i+1}\right)<k, \ldots, d\left(w_{i+r}, y_{r}\right)<k$ and the following holds. For every $j=i+1, \ldots, i+r, w_{j}$ lies in the unit sphere around $w_{j-1}$, where for convenience, we defined $w_{i}:=y_{i}$. It follows from the definitions that $x_{0}, \ldots, x_{i}, w_{i+1}, \ldots, w_{i+r}$ is a SAW and that $d\left(w_{i+r}, S_{1}\right)>\frac{r+3 \delta}{2}$. Now arguing as above, we see that we can find a suitable isometry $S$ of $\mathbb{H}^{d}$ such that $S\left(x_{i+r}\right)=w_{i+r}$ and

$$
d\left(S_{1}, S\left(S_{2}\right)\right)=d\left(w_{i+r}, S_{1}\right)-\frac{1-c}{2}>\frac{r+c-1+3 \delta}{2} .
$$

Now let $\mathcal{R}$ be the set of rotations around $w_{i+r}$ with the property that for every $R \in \mathcal{R}$, we have $d\left((R \circ S)\left(S_{2}\right), S_{1}\right)>3 \delta$ and $d\left((R \circ S)\left(S_{2}\right), w_{i+1}\right)>c, \ldots, d\left((R \circ S)\left(S_{2}\right), w_{i+r}\right)>c$. We claim that for every SAW $x$ and every $w \in W$, the set $\left\{(R \circ S)\left(x_{i+r+1}\right) \mid R \in \mathcal{R}\right\}$ has probability under the uniform measure in the unit sphere which is bounded away from 0 . Indeed, after applying a hyperbolic isometry, we can assume that $w_{i+r}$ coincides with the origin of $\mathbb{R}^{d}$. It is clear that the claim holds for Euclidean rotations around the origin. But a Euclidean rotation around the origin is also a hyperbolic one. This proves the claim.

It follows from the next lemma that all the SAWs constructed in this way satisfy $\mathcal{A}_{i}(\mathcal{C})$ for a certain constant $\mathcal{C}$. Consider now the conditional probability of $\mathcal{A}_{i}(\mathcal{C})$ given $x_{0}, \ldots, x_{i}$ and the relative position between the points $x_{i+r}, \ldots, x_{n}$ and notice that it is bounded away from 0 , whenever $x \in \mathcal{B}_{i, r}$. Taking expectation and using the fact that $r$ takes only finitely many values, we obtain the desired assertion.

We now prove the lemma mentioned above.

Lemma 2.3. Consider two bi-infinite geodesics $A, B$ in $\mathbb{H}^{d}$ with $d(A, B)>3 \delta$. Then for any $x_{0}, x \in A$ and $y_{0}, y \in B$ we have $d\left(\overline{x y}, \overline{x_{0} y_{0}}\right)<2 \delta$.

Proof. Let $x \in A, y \in B$ and write $\gamma, \gamma^{\prime}$ for the infinite geodesics that start at $x_{0}$ and contain $x, y$, respectively, parametrized by arc-length.

Consider some $R>0$ such that $d\left(\gamma(R), \gamma^{\prime}(R)\right)=2 \delta$. Notice that $\gamma^{\prime}(R) \in \overline{x_{0} y}$. We claim that $d\left(\gamma^{\prime}(R), \overline{x y}\right) \leq \delta$. Indeed, suppose to the contrary that $d\left(\gamma^{\prime}(R), \overline{x y}\right)>\delta$. Since $d\left(\gamma^{\prime}(R), \gamma \cup \overline{x y}\right)<\delta$, there exists some $t \in \gamma$ such that $d\left(\gamma^{\prime}(R), t\right)<\delta$. Hence $R-\delta<d\left(x_{0}, t\right)<R+\delta$, which implies that $d(\gamma(R), t)<\delta$. Thus $d\left(\gamma(R), \gamma^{\prime}(R)\right)<2 \delta$ and this contradiction proves the claim.

Now for the triangle with vertices $x_{0}, y_{0}, y$, we have that $d\left(\gamma^{\prime}(R), \overline{x_{0} y_{0}} \cup \overline{y_{0} y}\right)<\delta$. But $d\left(\gamma^{\prime}(R), \overline{y_{0} y}\right)>\delta$ because $d\left(\gamma(R), \gamma^{\prime}(R)\right)=2 \delta$ and $d(A, B)>3 \delta$. This shows that $d\left(\gamma^{\prime}(R), \overline{x_{0} y_{0}}\right)<\delta$ and completes the proof.

Using Lemma 2.2 and the fact that on any SAW of length $n$, the number of indices $i$ such that $\mathcal{B}_{i}$ occurs is of order $n$, we obtain that in a SAW of length $n$, the expected number of indices $i$, for which $x_{i}$ is within distance $\mathcal{C}$ to the geodesic between $x_{0}$ and $x_{n}$ is of order $n$. Since the open balls of radius $c / 2$ around the vertices of the SAW are disjoint, we get that the $\mathcal{C}$-neighbourhood of the geodesic has expected area of order $n$. But the area of the $\mathcal{C}$-neighbourhood of a geodesic of length $k$ is of order $k$ for any $k \geq 1$. This completes the proof of the theorem.

\section{Open problems}

In this section we will state some open problems. Given some $\varepsilon=\varepsilon(n)>0$, we consider continuous $n$ steps SAWs on $\mathbb{H}^{d}$, where now the steps are chosen uniformly from the sphere of radius $\varepsilon$ and we condition on sequences in which the distance between any pair of vertices is bigger than $c \varepsilon$, for some fixed $0<c<1$. We are interested in the behaviour of these SAWs as $\varepsilon$ tends to 0 . We will refer to the $n$ step model as the $(n, \varepsilon)$-SAW. Let us write $\mathbb{E}_{n, \varepsilon}$ for the expectation with respect to this measure. 
Conjecture 3.1. $\lim _{n \rightarrow \infty} \mathbb{E}_{n, 1 / n}\left(d\left(x_{0}, x_{n}\right)\right)=0$

Conjecture 3.2. There exists a constant $1>\beta=\beta(d, c)>0$ such that

$$
\lim _{n \rightarrow \infty} \mathbb{E}_{n, n^{-\beta}}\left(d\left(x_{0}, x_{n}\right)\right)
$$

exists and is not zero (possibly up to logarithmic factors).

We expect that $\beta$ is increasing in $c$ because when the self-avoidance restrictions are stronger, the walk tends to move further away from the origin.

Let us mention the observation motivating these conjectures. Given $\varepsilon>0$, let $\frac{1}{\varepsilon} \mathbb{H}^{d}$ denote $\mathbb{H}^{d}$ with the metric scaled by $\frac{1}{\varepsilon}$. It is clear that the $(n, \varepsilon)$-SAW on $\mathbb{H}^{d}$ coincides with the $(n, 1)$-SAW on $\frac{1}{\varepsilon} \mathbb{H}^{d}$. Moreover, $d\left(x_{0}, x_{n}\right)=\varepsilon d_{\varepsilon}\left(x_{0}, x_{n}\right)$, where $d_{\varepsilon}$ is the distance function on $\frac{1}{\varepsilon} \mathbb{H}^{d}$. As $\varepsilon$ tends to 0 , the curvature of $\frac{1}{\varepsilon} \mathbb{H}^{d}$ tends to 0 as well and $\frac{1}{\varepsilon} \mathbb{H}^{d}$ looks more and more like $\mathbb{R}^{d}$. This indicates that for certain values of $n$ and $\varepsilon$, the $(n, \varepsilon)$-SAW on $\mathbb{H}^{d}$ behaves like $(n, 1)$-SAW on $\mathbb{R}^{d}$. In fact, we can make a more precise prediction for the values of $n$ and $\varepsilon$ for which this holds. On balls of size smaller than $\frac{1}{\varepsilon}, \frac{1}{\varepsilon} \mathbb{H}^{d}$ looks similar to $\mathbb{R}^{d}$, while on balls of size larger than $\frac{1}{\varepsilon} \mathbb{H}^{d}$, the two spaces look different. If the $(n, 1)$-SAW on $\mathbb{R}^{d}$ is typically at distance $n^{\beta}$, then this indicates that the $(n, 1)$-SAW on $n^{-\beta} \mathbb{H}^{d}$ behaves like the $(n, 1)$-SAW on $\mathbb{R}^{d}$. This reasoning leads naturally to the above questions.

As $c$ tends to 0 , we expect that $\beta$ tends to $1 / 2$ because in the limit we obtain a random walk. Also, for $d \geq 5$, we expect that $\beta$ is always equal to $1 / 2$, as in this case, the scaling limit of SAW on $\mathbb{Z}^{d}$ is the Brownian motion [6].

\section{References}

[1] R. Bauerschmidt, H. Duminil-Copin, J. Goodman, and G. Slade, Lectures on self-avoiding walks. In Probability and Statistical Physics in Two and more Dimensions, 395-467, Clay Math. Proc. 15, Amer. Math. Soc., Providence, RI, (2012). MR-3059543

[2] I. Benjamini, Self avoiding walk on the seven regular triangulation. arXiv:1612.04169

[3] I. Benjamini and R. Eldan, Convex hulls in the hyperbolic space. Geom. Dedicata 160, (2012), 365-371. MR-2970060

[4] D. Burago, Y. Burago and S. Ivanov, A course in metric geometry. Graduate Studies in Mathematics 33 Am. Math. Soc., Providence, RI, (2001). MR-1835418

[5] H. Duminil-Copin and A. Hammond, Self-avoiding walk is sub-ballistic. Comm. Math. Phys. 324, (2013), 401-423. MR-3117515

[6] T. Hara and G. Slade, Self-avoiding walk in five or more dimensions I. The critical behaviour. Comm. Math. Phys. 147, (1992), 101-136. MR-1171762

[7] T. Hutchcroft, Self-avoiding walk on nonunimodular transitive graphs. Ann. Probab. 47, (2019), no. 5, 2801-2829. MR-4021237

[8] Z. Li, Positive speed self-avoiding walks on graphs with more than one end. J. Comb. Theory Ser. A. 175, (2020), 105257, 47 pp. MR-4094822

[9] N. Madras and C. Wu, Self-avoiding walks on hyperbolic graphs. Combin. Probab. Comput. 14, (2005), no. 4, 523-548. MR-2160417

[10] C. Panagiotis, Self-avoiding walks and polygons on hyperbolic graphs. arXiv:1908.00127 


\section{Electronic Journal of Probability Electronic Communications in Probability}

\section{Advantages of publishing in EJP-ECP}

- Very high standards

- Free for authors, free for readers

- Quick publication (no backlog)

- Secure publication $\left(\mathrm{LOCKSS}^{1}\right)$

- Easy interface (EJMS²)

\section{Economical model of EJP-ECP}

- Non profit, sponsored by $\mathrm{IMS}^{3}, \mathrm{BS}^{4}$, ProjectEuclid ${ }^{5}$

- Purely electronic

\section{Help keep the journal free and vigorous}

- Donate to the IMS open access fund ${ }^{6}$ (click here to donate!)

- Submit your best articles to EJP-ECP

- Choose EJP-ECP over for-profit journals

\footnotetext{
${ }^{1}$ LOCKSS: Lots of Copies Keep Stuff Safe http://www. lockss.org/

${ }^{2}$ EJMS: Electronic Journal Management System http://www.vtex.lt/en/ejms.html

${ }^{3}$ IMS: Institute of Mathematical Statistics http://www.imstat.org/

${ }^{4}$ BS: Bernoulli Society http://www. bernoulli-society.org/

${ }^{5}$ Project Euclid: https://projecteuclid.org/

${ }^{6}$ IMS Open Access Fund: http://www.imstat.org/publications/open.htm
} 